\title{
On the mobility of extended bodies in viscous films and membranes
}

\author{
Alex J. Levine \\ The Institute for Theoretical Physics, University of California, Santa Barbara CA 93106 and \\ Department of Physics, University of Massachusetts, Amherst Amherst MA 01060 USA * \\ T.B. Liverpool \\ The Institute for Theoretical Physics, University of California, Santa Barbara CA 93106 and \\ Department of Applied Mathematics, University of Leeds, Leeds LS2 9JT, England. I \\ F.C. MacKintosh \\ The Institute for Theoretical Physics, University of California, Santa Barbara CA 93106 and \\ Division of Physics \& Astronomy, Vrije Universiteit 1081 HV Amsterdam, The Netherland $\$$
}

(Dated: November 12, 2018)

\begin{abstract}
We develop general methods to calculate the mobilities of extended bodies in (or associated with) membranes and films. We demonstrate a striking difference between in-plane motion of rod-like inclusions and the corresponding case of bulk (three-dimensional) fluids: for rotations and motion perpendicular to the rod axis, we find purely local drag, in which the drag coefficient is purely algebraic in the rod dimensions. These results, as well as the calculational methods are applicable to such problems as the diffusion of objects in or associated with Langmuir films and lipid membranes. The methods can also be simply extended to treat viscoelastic systems.
\end{abstract}

\section{INTRODUCTION}

The motion of objects in biomembranes is important in many cellular processes. These objects are in many cases extended, macromolecular inclusions such as proteins 1, 2] or "rafts" [5, 6] of lipids. Thus, these can often be viewed as macroscopic objects moving in a continuum fluid environment. Studies of the motion of such inclusions in amphaphilic films [3, 4] and cell membranes have a long and interesting history. There are discrepancies in the early literature on protein diffusion in cell membranes, for instance, because of confusion over the applicability of three- versus two-dimensional diffusion to this case 7, 8]. One of the most important contributions in this area was that of Saffman [8], who noted that the two-dimensional motion in real membranes induces flows in the surrounding bulk (three-dimensional) fluid. He showed that the linearized Stokes-law does not describe the motion of inclusions in or bound to membranes. Rather, this represents a singular perturbation problem, and the drag coefficient is not a linear function of its size and the viscosity. In fact, the dependence is logarithmic, and thus the mobilities and diffusion coefficients of proteins in membranes are nearly independent of the object's size, in practice.

Furthermore, there is a length-scale determined by the ratio of membrane and fluid viscosities that determines the degree to which the dissipation is predominantly two- or three-dimensional [7, 8, 9, 10]. We call this length $\ell_{0}=\eta_{\mathrm{m}} / \eta_{\mathrm{f}}$, where $\eta_{\mathrm{m}, \mathrm{f}}$ are, respectively, the mem-

*levine@physics.umass.edu tannie@maths.leeds.ac.uk fcm@nat.vu.nl brane (two-dimensional) and fluid (three-dimensional) viscosities. A similar length, constructed from the twodimensional shear modulus and the fluid viscosity determines the scale of deformations controlled by in-plane versus fluid stresses in the case of elastic or viscoelastic membranes [11, 12, 13]. Here, we consider the motion of extended objects of large aspect ratio in such quasitwo-dimensional systems of, e.g., a viscous or viscoelastic membrane surrounded by a viscous solvent. In such cases, both short- and large-scale dissipation can play a role. We examine in detail the motion of rod-like inclusions, but we present a general scheme for the calculation of the translational and rotational mobilities of arbitrary extended bodies.

Even for the case of the motion of simple, rod-like objects in a bulk Newtonian fluid (i.e., in three dimensions), the situation is subtle [7, 14, 15]. For instance, for either infinitely long rods in three dimensions or for the motion of point-like objects in two dimensions, there is no true low Reynolds number regime (i.e., linear hydrodynamics) [Batchelor 1967]. Specifically, there is no linear drag coefficient for such a system: the drag force does not depend linearly with velocity. For finite length rods and small enough velocities, however, there is a linear drag coefficient. The drag coefficients for motion parallel and perpendicular to the rod axis are given by

$$
\zeta_{\perp}=2 \zeta_{\|}=4 \pi \eta / \ln (A L / a)
$$

per unit length, where $L$ is the rod length, $a$ is its radius, and $A$ is a constant of order unity, depending on the precise geometry.

We examine here a variant of this hydrodynamic problem, in which the rigid rod lies in a two-dimensional interface that is viscously coupled to a bulk, Newtonian liquid phase. The generalization of this problem to the motion of such a rod embedded in a viscoelastic film is 
straight-forward. There are many physical realizations of motion in viscous and viscoelastic films. These include, for instance, the motion of extended, membraneassociated proteins in or on the surface of lipid bilayers 1, 2]. Our work here is also motivated by recent experiments that have demonstrated the possibility of constructing and making quantitative measurements on viscoelastic films that closely resemble cellular structures such as the actin cortex [11]. Driven motion of rods in viscous/viscoelastic films has also been used to determine rheological properties, e.g., of monolayers [18]. In addition, the understanding of this problem will allow the quantitative interpretation of the thermally excited angular fluctuations of microscopic rod-like particles (such as fd virus) embedded in such systems. Such hydrodynamic studies may also shed light on the dynamics of inclusions and transmembrane protein complexes in fluid cell membranes. Furthermore, we note that calculational methods developed in this work allow one to compute the hydrodynamic drag of irregularly shaped objects embedded in the film. Such objects can be e.g. fractal aggregates [16], or lipid rafts in call membranes.

We find two principal results: (i) for small objects (specifically, for which $L \ll \ell_{0}$ ), the drag coefficients become independent of both the rod orientation and aspect ratio; and (ii) for larger rods of large aspect ratio, $\zeta_{\perp}$ becomes purely linear in the rod length $L-i$.e., the drag becomes purely local. In contrast, we find that the wellestablished three-dimensional result $\zeta_{\|}=2 \pi \eta / \ln (A L / a)$ applies for parallel motion in the film, provided that $L \gg \ell_{0}$. Here, however, the effective rod radius becomes $\ell_{0}$ rather than the physical radius $a$, when $a \ll \ell_{0}$. Closely related to (ii), we find that the rotational drag (equivalently diffusion constant) depends purely algebraically on the rod length.

We consider only the most simple class of rod geometries in that we assume the rod to have a circular cross section in the plane perpendicular to its axis. We take the radius of that circle to be $a$ while the length of the rod is given by $L$. The geometry of this rod of length $L$ and radius $a$ is then parameterized by only one dimensionless ratio, the aspect ratio $\rho$ defined by

$$
\rho=\frac{L}{2 a} .
$$

The long axis of the rod may be assumed to be terminated by spherical end caps although, as will be seen below, our calculation is not sensitive to the fine structure of the ends due to our introduction of a short distance cutoff in the problem. We will argue below, however, that for the case of large aspect ratio rods, the detailed structure of the end caps will have a negligible effect on the overall drag.

The fundamental distinction between the well-known result for the hydrodynamic drag on a rod in a homogeneous, three-dimensional viscous material and our result for the drag on a rod embedded in a viscous membrane coupled to a viscous, three-dimensional fluid is the ap- pearance of the length scale $\ell_{0}$. This length enters since the ratio of a two-dimensional interfacial viscosity $\eta_{\mathrm{m}}$ and the usual viscosity $\eta_{\mathrm{f}}$ of the bulk fluid has dimensions of length,

$$
\ell_{0}=\frac{\eta_{\mathrm{m}}}{\sum \eta}
$$

The denominator, $\sum \eta$ is the sum of the viscosities of the fluid above the interface (the superphase) and fluid below the membrane (the subphase), in the general case of two different viscosities (or one vanishing one, in the case of Langmuir films). For a membrane embedded in a uniform fluid of viscosity $\eta$, this is $\sum \eta=2 \eta$. In general, however, we may have two distinct nonzero viscosities. For a Langmuir monolayer we have $\sum \eta=\eta$, since we can neglect the viscosity of air. From here on, however, we shall simply use the length $\ell_{0}$ and $\eta_{\mathrm{f}}$ for the sum of the viscosities of the two bulk fluids surrounding the membrane. Throughout this paper we measure all lengths in terms of the fundamental length $\ell_{0}$, except where explicitly stated to the contrary.
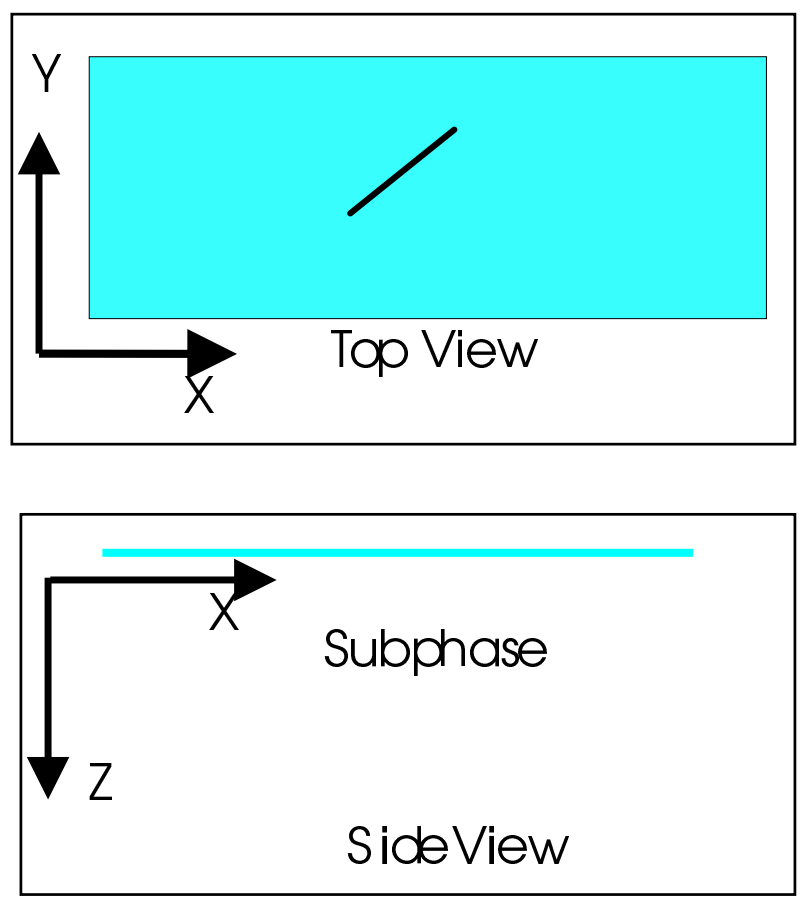

FIG. 1: The upper figure shows the film from the top looking down. The rod is shown as the black line and the film is shown in blue. The rod is assumed to be embedded in the film, but not in the bulk subphase below it. This subphase is shown in the lower figure, which pictures the system from a side view. In this paper the Newtonian subphase is assumed to be infinitely deep.

There are three independent drag coefficients or particle mobilities to determine in the problem. The mobility tensor is the inverse of the drag coefficient tensor. For translational motion, the mobility tensor $\mu_{i j}$ of the rod 
in the membrane is defined by:

$$
v_{i}^{\text {rod }}=\mu_{i j} F_{j},
$$

where $v_{i}^{\text {rod }}$ is the $i^{\text {th }}$ component of the velocity of the rod and $F_{j}$ is the $j^{\text {th }}$ component of the force applied to the rod. By in-plane rotational symmetry combined with the $\hat{n} \longrightarrow-\hat{n}$ symmetry of the rod, the mobility tensor must take the form:

$$
\mu_{i j}=\mu_{||} \hat{n}_{i} \hat{n}_{j}+\mu_{\perp}\left(\delta_{i j}-\hat{n}_{i} \hat{n}_{j}\right) .
$$

Here $\mu_{\perp}$ and $\mu_{\|}$are the mobility of the rod dragged perpendicular to and parallel to its long axis respectively. In addition to these two independent translational mobilities, there is also one rotational mobility, $\mu_{\text {rot }}$ linking the angular velocity of the rod to the torque applied to that rod about its center of inversion symmetry.

It should be noted that there is no hydrodynamic torque acting on the rod when it is dragged by any force acting through the center of inversion symmetry of the rod. We refer to these forces as "central." This can be seen from the following argument: Torque in the twodimensional plane is a pseudoscalar that must be odd under time reversal. Such a pseudoscalar can only be built out of the antisymmetric combination of velocity vector of the rod $\mathbf{v}$ and a vector along the axis of the rod $\hat{\mathbf{n}}$. That combination $\epsilon_{\alpha \beta} v_{\alpha} \hat{n}_{\beta}$ must also be symmetric under $\hat{\mathbf{n}} \longrightarrow-\hat{\mathbf{n}}$ since the rod is symmetric upon such transformation. Thus the only available pseudoscalar is disallowed. Therefore there is no rotational motion generated by this class of central forces and the rotational degree of freedom decouples from the two translational degrees of freedom. We further note that boundary conditions that break rotational symmetry or the application of a force at a point other than the center of symmetry violate the above assumptions and thus allow a coupling of the rotational and translational motion of the rod.

We approach the solution of this problem by two complimentary means. In the first part of the calculation, section IIA we approximate the continuous rod by a series of discs, in analogy to the Kirkwood approximation used in the calculation of the drag on a rod in a uniform, three-dimensional viscous environment [15]. This calculation allows one to incorporate the details of the shape of the rod in the resulting drag coefficient. Here the shape of the rod is parameterized in terms of its dimensionless aspect ratio. This method, however, becomes numerically difficult in the limit of large aspect ratios, i.e., for very long, thin rods. In this limit we may proceed by a second approximation that assumes the rod to be infinitely thin-i.e., $L \gg a$. Here, we also restrict our attention to the limit $a \ll \ell_{0}$.

The outline of the remaining parts of this paper are as follows: In section $1 \mathrm{~A}$ we develop the first of two calculational methods for determining the drag on rodlike objects embedded in the membrane. Then in section IIB we demonstrate a second approach to determine the drag on a rod. This approach is optimized to work in the limit of high aspect ratio rods and compliments the first approach which is most efficient for rods of small aspect ratio, i.e., less elongated objects. The reader who wishes to examine the results of these calculations without delving into their details may skip to section[II] where the drag coefficients for translational motion parallel and perpendicular to the long axis of the rod are computed for a variety rod geometries. In addition we explore the rotational drag on these rods there. Finally, we conclude and discuss our results in section IV]

\section{THE RESPONSE FUNCTION}

\section{A. By the Kirkwood approximation}

To incorporate the correct dynamics of this coupled system, we use the results of our previous calculation [19] for the velocity response of the membrane at a position $\mathbf{x}$ due to the application of a force localized at the origin. Specializing to the case of a purely viscous (as opposed to a viscoelastic) film, we can write the membrane velocity response to a point force localized at $\mathbf{x}^{\prime}$ using the previously defined response function:

$$
v_{\alpha}(\mathbf{x})=\alpha_{\alpha \beta}\left(\mathbf{x}-\mathbf{x}^{\prime}\right) f_{\beta}\left(\mathbf{x}^{\prime}\right)
$$

in a closed form as

$$
\alpha_{\alpha \beta}(\mathbf{x})=\alpha_{\|}(|\mathbf{x}|) \hat{x}_{\alpha} \hat{x}_{\beta}+\alpha_{\|}(|\mathbf{x}|)\left[\delta_{\alpha \beta}-\hat{x}_{\alpha} \hat{x}_{\beta}\right],(6)
$$

where the scalar functions $\alpha_{\|}, \alpha_{\perp}$ of the distance between the point of the force application and the measurement of the velocity field are given by:

$$
\begin{aligned}
& -i \omega \alpha_{\|}(x, \omega)= \\
& \frac{1}{4 \pi \eta_{m}}\left[\frac{\pi}{z} \mathbf{H}_{1}(z)-\frac{2}{z^{2}}-\frac{\pi}{2}\left(Y_{0}(z)+Y_{2}(z)\right)\right]
\end{aligned}
$$

and

$$
\begin{aligned}
& -i \omega \alpha_{\perp}(x, \omega)= \\
& \frac{1}{4 \pi \eta_{m}}\left[\pi \mathbf{H}_{0}(z)-\frac{\pi}{z} \mathbf{H}_{1}(z)+\frac{2}{z^{2}}-\frac{\pi}{2}\left(Y_{0}(z)-Y_{2}(z)\right)\right],
\end{aligned}
$$

where the $\mathbf{H}_{\nu}$ are Struve functions 20], and the $Y_{\nu}$ are Bessel functions of the second kind. We note $z=|\mathbf{x}| / \ell_{0}$ is the distance between the point of force application and the membrane velocity response measured in the flat membrane in units of $\ell_{0}$. At this point, it is important to distinguish between the two response functions introduced: the mobility tensor of the rod $\mu_{i j}$ gives the velocity response $v_{i}$ of the rod given a total force $F_{j}$ acting on it through its center. The response function $\alpha_{i j}\left(\mathbf{x}-\mathbf{x}^{\prime}\right)$ gives the velocity response $v_{i}(\mathbf{x})$ of the two-dimensional fluid at the position $\mathbf{x}$ due the application of a point force $F_{j}\left(\mathbf{x}^{\prime}\right)$ at another point $\mathbf{x}^{\prime}$ in the two-dimensional fluid. The main purpose of this and the next section of the paper it to derive the former response function for the rod 
from the latter response function for the fluid, which we have previously calculated [19].

We note also that, due to the linearity of the underlying low-Reynolds number hydrodynamics, the velocity response produced at some point in the membrane by a collection of point forces is simply the sum of the response functions appropriate to each point force individually:

$$
v_{\alpha}(\mathbf{x})=\sum_{n=1}^{N} \alpha_{\alpha \beta}\left(\mathbf{x}-\mathbf{x}_{n}\right) f_{\beta}\left(\mathbf{x}_{n}\right)
$$

where $n$ indexes the $N$ point forces located respectively at locations $\mathbf{x}_{n}$ in the film. Clearly the sum may be converted into an integral for case of a continuous force distribution; we will examine this limit for the case of an infinitely thin rod of finite length in the next section.

Using this superposition principle we may determine the effective drag on a rod by employing the twodimensional analogue of the Kirkwood approximation used to calculate the hydrodynamic drag on a rod in three dimensions. Specifically, we replace the rod of length $L$ and cross-sectional radius $a$ by a set of $n+1$ disks of radius $a$ and intersphere separation $b$ chosen so that the total length of the rod is preserved, 1.e. $L=n b+2 a$. See figure 2 In this way, the aspect ratio of the rod, $\rho=L / a$ can be fixed. The number of disks, of course, can be varied, however, we will always choose that number to be the maximal number consistent with a given aspect ratio and the non-interpenetrability of the disks. We have also shown that computed hydrodynamic drag on the rod is only weakly dependent on the number of disks (or equivalently on the inter-disk separation).

Our strategy for computing the drag on the rod involves setting the rod in uniform motion with unit velocity by imposing some set of forces $\mathbf{f}^{(i)}, i=1, \ldots, n+1$ on the $n+1$ disks that make up the rod. Clearly the total force on the rod, which is equal to the effective drag coefficient is simply the sum of those forces:

$$
F_{\alpha}=\sum_{i=1}^{n+1} f_{\alpha}^{(i)}=\zeta_{\alpha \beta} v_{\beta}
$$

Using Eq. (9) we can compute the velocity field for a given collection of point forces. However, to determine the effective drag on the rod, one needs to insist that all the beads have the same given velocity and determine the forces applied to them to ensure this result.

For definiteness, we first discuss the drag on the rod when moving perpendicularly to its long axis. We may then directly write out the analogous solution for motion parallel to the long axis of the rod. Motion of the rod along any arbitrary direction in the plane relative to its long axis can be obtained from these two results using the linearity of problem. Thus these solutions span all possible linear motions of the rod. We will return to rotational motion shortly.

To calculate the drag on the rod moving perpendicular to its long axis (see figure 1), we first apply Eq. (9) to
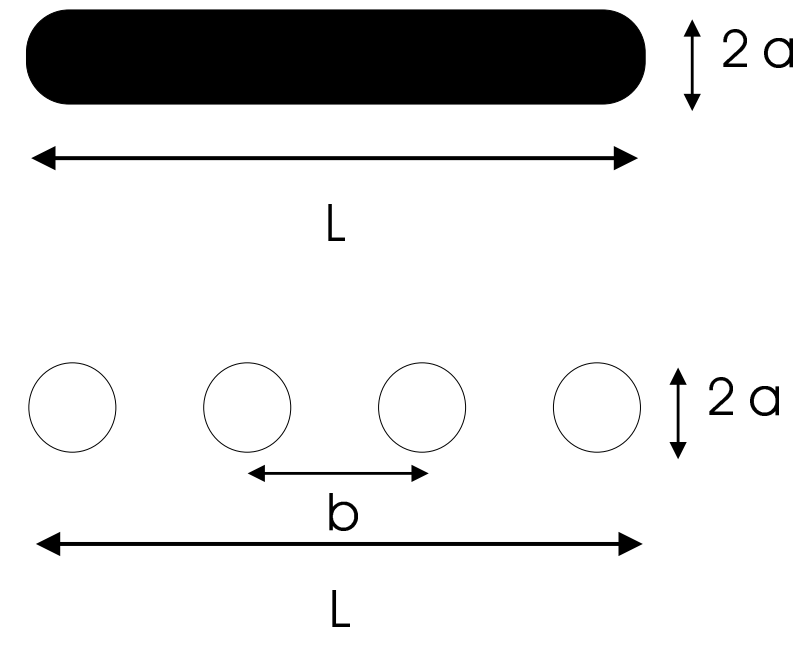

FIG. 2: Approximating the continuous rod by a series of balls of radius $a$ with center-to-center separation $b$. The number of balls and their separation is chosen so that collection of balls has the same length $L$ as the original rod. For the best approximation to the original rod, we maximize the number of balls for a given aspect ratio of the original rod.

determine the velocity of all $n+1$ spheres making up the rod as a function of as yet unknown forces. We insist only, based on the symmetry of this problem, that these forces are also perpendicular the rod's axis. Thus,

$$
v^{(i)}=\sum_{i=1}^{n+1} \alpha_{\perp}^{(i j)} f^{(j)}
$$

where we have suppressed the vectorial indices, defined $v^{(i)}$ to be the velocity of the $i^{\text {th }}$ sphere at position $\mathbf{x}^{(i)}$, and rewritten the mobility tensor using Eq. (8) and the definition:

$$
\alpha_{\perp}^{(i j)}=\alpha_{\perp}\left(\mathbf{x}^{(i)}-\mathbf{x}^{(j)}\right)
$$

The solution for the forces on the individual beads and thus, using Eq. (10), the total force on the rod and equivalently the hydrodynamic drag is then obtained by inverting the $(n+1) \times(n+1)$ matrix of response functions $\alpha_{\perp}^{(i j)}$. We call this inverse matrix $\mathcal{M}^{i j}$ defined by:

$$
\mathcal{M}_{\|, \perp}^{(i j)}=\left(\alpha_{\|, \perp}^{-1}\right)^{(i j)}
$$


The drag coefficient is then

$$
\zeta_{\perp}=\sum_{i, j=1}^{n+1} \mathcal{M}_{\perp}^{(i j)}
$$

where we have used Eq. (10) and the fact that each bead making up the rod has a velocity of unity. The analogous expression from the drag on the rod moving parallel to its long axis is then obtained by replacing the terms of the mobility matrix, $\mathcal{M}_{\perp}^{(i j)}$, with $\mathcal{M}_{\|}^{(i j)}$ since here all the bead velocities are parallel to the separation vectors between the beads. We then have

$$
\zeta_{\|}=\sum_{i, j=1}^{n+1} \mathcal{M}_{\|}^{(i j)}
$$

and the complete solution for motion of the rod in the plane at an arbitrary angle $\phi$ with respect to its long axis (see figure 11) is given by

$$
\zeta(\phi)=\zeta_{\|} \cos \phi+\zeta_{\perp} \sin \phi .
$$

It is perhaps not surprising that the drag on the rod does not have a simple, closed-form solution in the general case. Here we examine the results of a numerical calculation of the these drag coefficients. The results depend on two parameters reflecting the relative magnitudes of three lengths involved in the problem. We report our results in terms of the two dimensionless numbers introduced above: the overall length of the rod (i.e., the linear dimension of its long axis) measured in units of $\ell_{0}$, $L / \ell_{0}$, and the aspect ratio of the $\operatorname{rod} L / a$.

\section{B. Thin rod approximation}

As a calculation tool, the above Kirkwood approximation has one shortcoming. If one wishes to explore the drag on a very thin rod compared to its length,i.e., one of high aspect ratio, one is compelled to use a large number of disks. In fact, the number of disks is linearly proportional to the aspect ratio of the rod being modelled. Since even numerically inverting a large matrix is cumbersome, the penultimate step in the procedure outlined above becomes slow in the high aspect ratio limit. Fortunately, there is a complementary approach to the Kirkwood scheme that involves inverting a more manageable matrix and is still valid for exploring rods of infinite aspect ratio, or specifically, rods of finite length, but vanishing thickness. Since it is precisely in this limit of large aspect ratio that the Kirkwood approximation becomes intractable, the combination of these two approaches allows us to study the drag on rod for both large and small aspect ratios.

The thin rod approximation starts from the assumption that one can take the limit of an infinitely thin rod from the start. Thus the velocity field at the point $\mathbf{x}$ due to a continuous distribution of force densities along the rod, $\mathbf{f}(x \hat{x})$ that lies along the $\hat{x}$-axis from $x=-L / 2$ to $x=L / 2$ takes the form

$$
v_{i}(\mathbf{x})=\int_{-L / 2}^{L / 2} \alpha_{i j}(\mathbf{x}-p \hat{x}) f_{j}(p \hat{x}) d p
$$

the indices in the above equation represent the usual vectorial indices; there are no disks to count here. There has been a simplification introduced in Eq. 17. The integral over the force density, $f(\mathbf{x})$ which in principal extends over the entire volume of the rod has been made onedimensional by assuming the rod to be infinitely thin. This simplification is possible since the short distance behavior of the response function includes only an integrable singularity.

After writing Eq. 17 one is still faced with the problem of inverting the equation. After all, the assumed rigidity of the rod requires that $\mathbf{v}(\mathbf{x})$ be constant along the rod, but force on any length element of the infinitely thin rod are unknown as they are comprised of both the externally applied for and, as yet undetermined internal forces of constraint. Our method of solution is fundamentally identical to that used in the previous section, we impose a unit velocity field on the rod and determine the force density required to affect his result, $\mathbf{f}(\mathbf{x})$. We use this intermediate result by integrating the linear force density over the length of the rod to find the total force necessary to move the rod with a velocity of unity. This force is clearly just the drag coefficient that we sought.

The inversion of Eq. 17 proceeds by first expanding the linear force density in Legendre polynomials $P_{n}(2 x / L)$. From symmetry considerations we can expand the force density in even Legendre polynomials, i.e.,

$$
f(x)=\sum_{n=0}^{N} c_{2 n} P_{2 n}(2 x / L)
$$

for the both the perpendicular and parallel dragging calculations, while we expand the force density in odd Legendre functions to study the rotational drag on the object. In what follows, we only describe in detail the case of linear drag, although the rotational case is very similar. The coefficients $c_{n}$ are as yet unknown, since the function $f(x)$ is undetermined. In principle since the Legendre polynomials form a complete set on the interval -1 to +1 , any physical force density can be expressed as in Eq. 18 provided $N$ be taken to infinity. In practice we find excellent numerical results even when truncating this sum to just the first few terms, taking into account the symmetry of the force distribution about the center of the rod. We discuss in more detail below the meaning of numerical accuracy as used in the present context.

Using the expansion of the force density from Eq. 18 we can rewrite Eq. 17 as

$$
\mathbf{v}(\mathbf{x})=\sum_{n=0}^{N} c_{2 n} \int_{-L / 2}^{L / 2} \alpha(\mathbf{x}-z \hat{x}) P_{2 n}(2 z / L) d z
$$


To further simplify the analysis, we relax the requirement that the velocity field be equal to unity at all points along the rod. Rather we impose this condition at a finite set of points $0 \leq p_{i} \leq L / 2$ along the rod. Given that we intend to truncate the Legendre function expansion of the force density at $N$, we can impose the velocity condition at a maximum of $N+1$ points without creating an overdetermined system of equations. Thus we require that:

$$
\begin{aligned}
\mathbf{v}\left(p_{i} \hat{x}\right) & =0, \text { for } i=0, \ldots, N \\
1= & \sum_{n=0}^{N} c_{2 n} \int_{-L / 2}^{L / 2} \alpha\left(\hat{x} p_{i}-z \hat{x}\right) P_{2 n}(2 z / L) d z \\
& \quad \text { for } i=0, \ldots, N .
\end{aligned}
$$

Now finding the force distribution along the rod requires only the inversion of an $(N+1) \times(N+1)$ matrix, $\mathcal{N}_{i j}$ whose components are defined by

$$
\mathcal{N}_{i j}=\int_{-L / 2}^{L / 2} \alpha\left(\hat{x} p_{i}-z \hat{x}\right) P_{2 j}(2 z / L) d z .
$$

Finally, the total force on the rod is found by reconstructing the force density from its Legendre polynomial expansion and integrating the resulting expression over the entire rod. That force density is given by Eq. 18 where the coefficients, $c_{k}$ are determined by solving the set of equations Eqs. 20] using the inverse of the matrix defined in Eq. 22 Thus we find

$$
c_{k}=\sum_{i=0}^{N} \mathcal{N}_{k i}^{-1} .
$$

Due to the orthonormality of the Legendre polynomials, the total force is given entirely by the coefficient of the zeroth Legendre polynomial, $c_{0}$. Similarly, the total torque in the case of rotations is given by the coefficient of the first odd Legendre polynomial.

There remains one more aspect to this calculation. How does one determine the accuracy of the approximation and how can one optimize that accuracy by selected the set of points along the $\operatorname{rod} x_{i}, i=0, \ldots, N$ at which to impose the velocity boundary condition? We first judge the accuracy of the result by calculating the velocity field along the rod. Ideally the value of the velocity should be unity. Because we have only imposed that boundary condition at a discrete set of points along the rod, the velocity field along the rod varies. We find that with only eleven points chosen symmetrically about the center of the rod (i.e., $N=5$ above), the velocity field deviates from unity by at most five percent in most cases. However, we find that the drag coefficient (defined by the ratio of total force to average velocity) converges much more rapidly with $N$. Specifically, we find a variation of less than a percent in the drag coefficient in all cases, so long as $N>2$. Below, we report our results for $N=5$. We thus are able to achieve better than one percent accuracy with only a $6 \times 6$ matrix.

\section{RESULTS}

Figure 3 shows the parallel drag coefficient for a rod as a function of its length, $L / \ell_{0}$ in reduced units. Two finite aspect ratios, as well as the case of infinite aspect ratio, i.e., an infinitely thin rod, are shown in this figure. As expected based on the above discussion, the shape of the rod and thus its aspect ratio does not effect the drag on the rod in the limit all dimensions of the rod are small compared to $\ell_{0}$. Essentially all small rod-like particles behave in the membrane as if they were infinitely thin. For rods longer than this crossover length, the shape of the rod clearly affects its hydrodynamic drag as seen by the deviation of the finite-aspect-ratio curves from the $\rho=\infty$ curve shown as the solid line in the figure. It should be noted that the finite aspect ratio calculations were performed using the Kirkwood approximation, while the drag on the infinitely thin $\operatorname{rod}(\rho=\infty)$ is calculated using the thin-rod approximation.

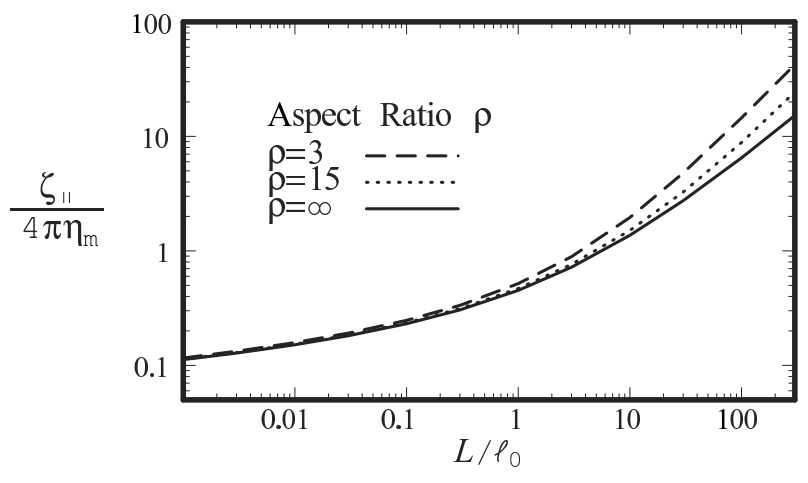

FIG. 3: Parallel drag coefficient of a rod for various aspect ratios.

The analogous calculation can be made for the drag on the rod moving in a direction perpendicular to its long axis. These results are shown in figure 4

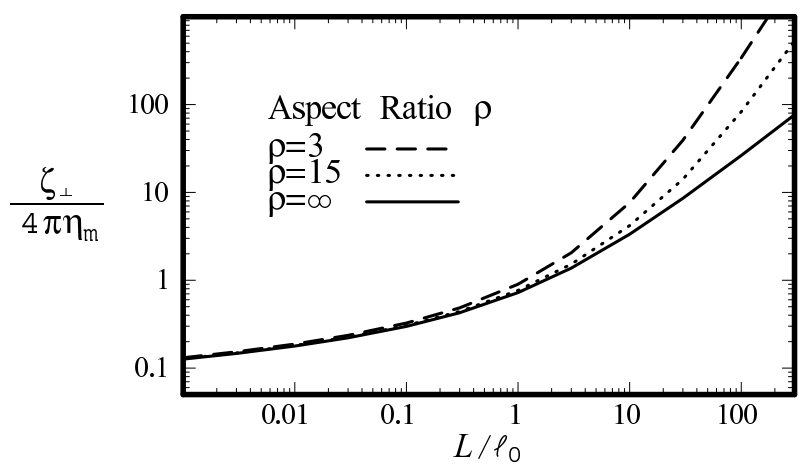

FIG. 4: Perpendicular drag coefficient of a rod for various aspect ratios.

The convergence of the finite aspect ratio rod results 
towards the infinite aspect ratio result clearly tests the consistency of the two numerical approaches to drag calculation. To study in more detail the aspect ratio dependence of the drag we plot the drag coefficient for parallel motion (figure 5] and perpendicular motion (figure 6).

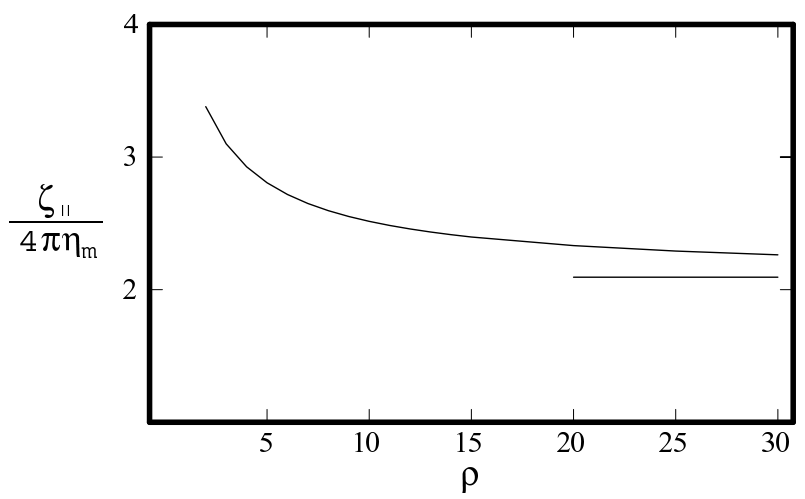

FIG. 5: The parallel drag coefficient of a finite aspect ratio rod as a function of aspect ratio, $\rho$. The result for an infinite aspect ratio is shown as a horizontal line. The length of the $\operatorname{rod}$ is $20 \ell_{0}$.

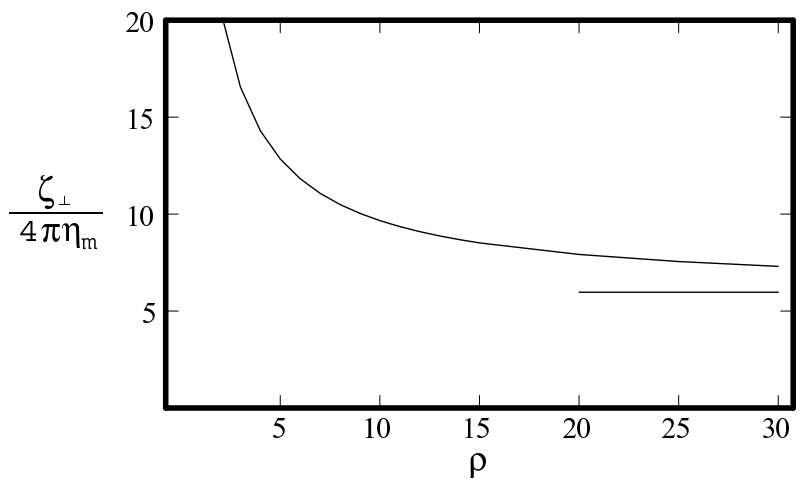

FIG. 6: The perpendicular drag coefficient of a finite aspect ratio rod as a function of aspect ratio, $\rho$. The result for an infinite aspect ratio is shown as a horizontal line. The length of the rod is $20 \ell_{0}$

In both of these figures 5 and 6 the length of the rod was held constant so that $L / \ell_{0}=20$. As discussed above particle shape is less relevant for particles with dimensions less than $\ell_{0}$ so a rod of significantly longer length was chosen to explore the aspect ratio dependence of the rod's drag coefficient. To observe the importance of the particle size (measured in the natural units of $\ell_{0}$ ) we plot the aspect ratio dependence of the drag coefficient of a rod of length $0.1 \ell_{0}$. The drag coefficient of the rod moving parallel to its long axis is shown in figure 7 while the drag coefficient of the rod moving perpendicular to its long axis is shown in figure 8

It may be observed from a comparison of the pairs of corresponding figures for parallel drag and perpendicular drag such as 7 vs. 8 , 5. 6s. 6, and 3 vs. 4 that the perpendicular drag coefficient is strictly larger than the par-

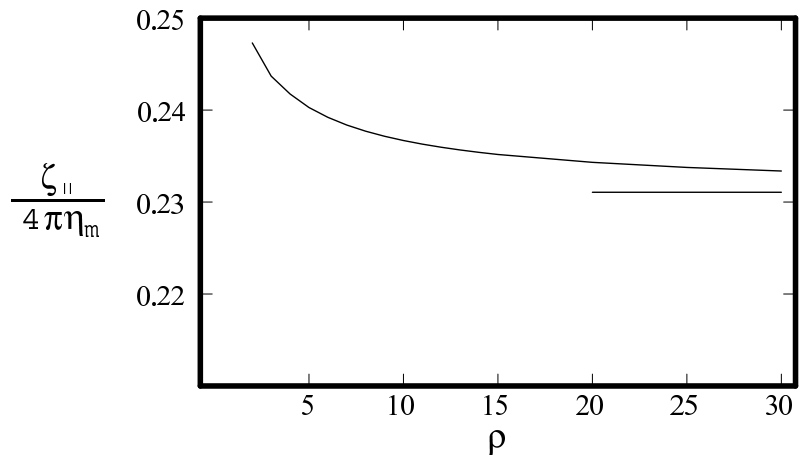

FIG. 7: The parallel drag coefficient of a finite aspect ratio rod as a function of aspect ratio, $\rho$. The result for an infinite aspect ratio is shown as a horizontal line. The length of the $\operatorname{rod}$ is $0.1 \ell_{0}$

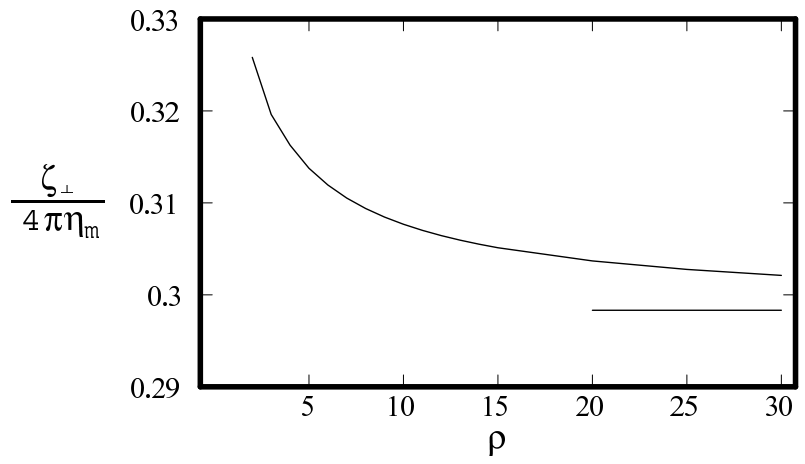

FIG. 8: The perpendicular drag coefficient of a finite aspect ratio rod as a function of aspect ratio, $\rho$. The result for an infinite aspect ratio is shown as a horizontal line. The length of the rod is $0.1 \ell_{0}$

allel drag coefficient for rods of all aspect ratios (greater than one, i.e. not disks) and lengths. The magnitude of the difference between these two drag coefficients, however, depends on the length of the rod compared with the natural length, $\ell_{0}$. For lengths such that $L<\ell_{0}$, the two drag coefficients converge to the same value and the difference between these coefficients grows monotonically with increasing rod length. As an example, the two drag coefficients are plotted as a function of rod length for infinite aspect ratio rods in figure 9]

The two coefficients begin to separate at rod lengths on the order of $\ell_{0}$. This differs not only from the small rod limit but also from the case of motion in bulk fluids, where the two drag coefficients differ only by a constant factor of two. The length dependence here can be understood by noting that $\ell_{0}$ sets the natural length scale over which the two-dimensional fluid velocity field can vary. In detail, what we find is that the parallel drag in the film is essentially unchanged from the bulk, three-dimensional drag, in that

$$
\zeta_{\|}=\frac{2 \pi \eta L}{\ln \left(0.43 L / \ell_{0}\right)},
$$




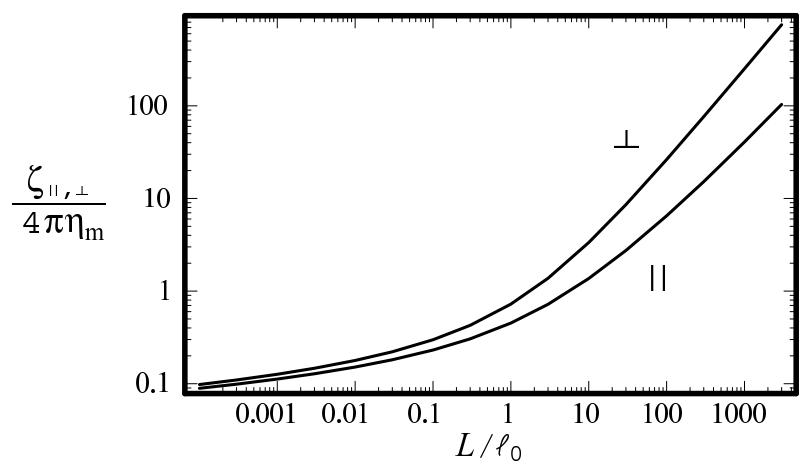

FIG. 9: A comparison of the perpendicular and parallel drag coefficients as a function of rod length for a rod of infinite aspect ratio. Note that the difference between the two coefficients is a monotonically increasing function of rod length and that the two coefficients begin to diverge at the length $L \simeq \ell_{0}$.

where the prefactor in the logarithm has been determined to within $1 \%$. Comparing with the result for drag of a rod in a bulk fluid, the effective radius of the rod is now of order $\ell_{0}$ (for $a \ll \ell_{0}$ ). On the one hand, that the three-dimensional result is recovered is not surprising, given that $\ell_{0}$ corresponds physically to a length scale beyond which the fluid viscosity dominates the film viscosity. Furthermore, the corresponding fluid velocity field in this case both respects the assumed incompressibility of the film, and is the same as that of rod motion in a fluid above this length $\ell_{0}$. Thus, $\ell_{0}$ determines the effective aspect ratio. The case of perpendicular motion, on the other hand, is qualitatively very different. We find

$$
\zeta_{\perp}=2 \pi \eta L .
$$

Here, the corresponding bulk fluid velocity field in the absence of the film is inconsistent with incompressibility of the film. Specifically, there is a non-vanishing twodimensional divergence of velocity field restricted to the plane of motion for motion perpendicular to the rod axis. Hence, although only the bulk fluid viscosity $\eta$ enters this expression (to be expected since dissipation is dominated at the largest scales by the fluid viscosity), the in-plane incompressibility requires that the fluid velocity field extends over distances comparable to the largest dimension $L$. This means that the usual hydrodynamic coupling of portions of the rod (represented by the logarithm) is not present. The result is a drag coefficient purely linear in rod length. In other words, the drag is effectively local in character.

Finally, we note the result for the rotational drag coefficient on the rod which gives the required torque applied to rod about the center to generate an angular velocity of the rod equal to unity. As discussed in the previous sections, this calculation proceeds analogously to those of the perpendicular and parallel drag coefficients. We plot the rotational drag coefficient divided by $L^{2}$ for a rod of infinite aspect ratio as a function of the reduced length

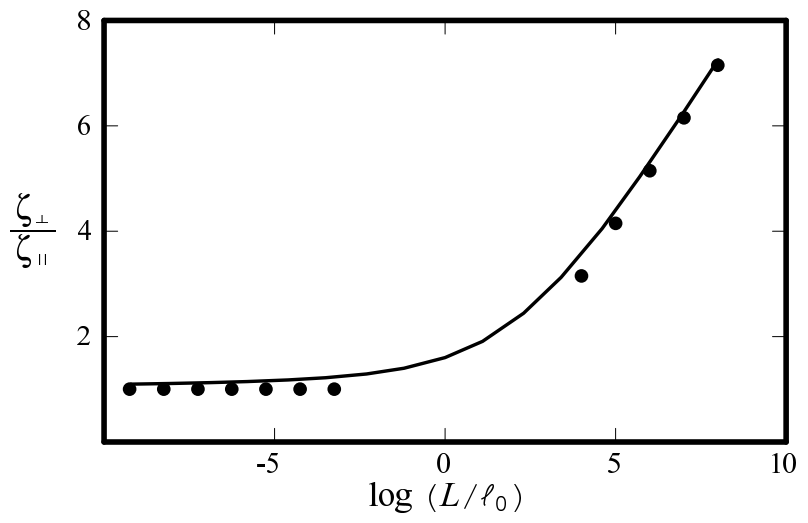

FIG. 10: The solid line is the ratio of the perpendicular drag coefficient to the parallel drag coefficient calculated in the thin rod approximation. The dotted lines are two different asymptotic fits to this curve corresponding to short rods and long rods. For short rods this fit is to the constant one consistent with the Saffman-Delbrück result. The large rods fit is to a simple logarithm in length as discussed in the text.

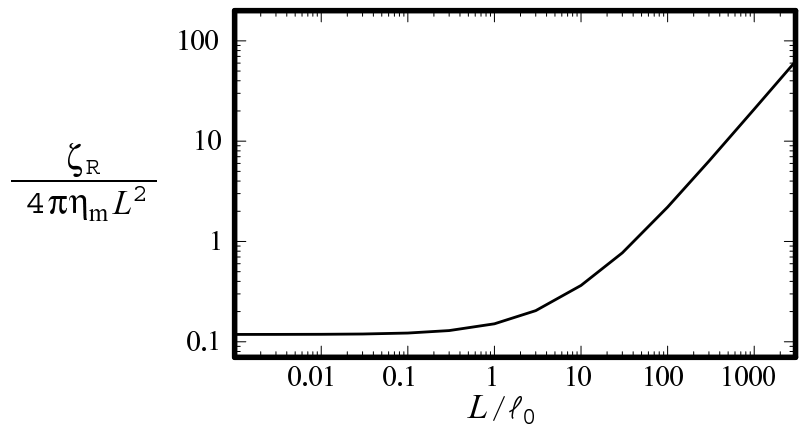

FIG. 11: The rotational drag coefficient of a rod of infinite aspect ratio plotted versus the length of the rod.

in figure 11] The essential feature of this plot is that rotational drag coefficient scales as $L^{2}$ for rods smaller than $\ell_{0}$ and then as $L^{3}$ for rods longer than this natural length. Thus, we find purely algebraic behavior in both limits.

\section{SUMMARY}

Using the response function previous calculated [19] we have calculated the hydrodynamic drag on a rod moving at low Reynolds number in a viscous film coupled to fluid sub- and superphases of arbitrary viscosity. The drag coefficient on the rod is a tensorial object with two independent parameters that correspond to the drag coefficient of the rod moving along its long axis (parallel) and in the direction perpendicular to its long axis. We have also computed the rotational drag coefficient.

These results were calculated numerically using two methods with complementary regimes of validity; the Kirkwood method, which approximates the rod as a se- 
ries of non-interpenetrating disks linked together and is well suited to calculating the drag coefficients for rods of smaller aspect ratio. The aspect ratio is set by choosing the number of these non-interpenetrating disks to make up the rod. For very long, thin rods having higher aspect ratios, this method becomes numerically cumbersome since it involves inverting an $n \times n$ matrix for a rod made up of $n$ disks and reaching higher aspect ratios requires adding more disks. To explore the limit of very high aspect ratios, one can perform calculations in the infinite aspect ratio limit. These two methods can be shown to be consistent numerically; in the limit of a large number of disks, we have checked numerically that the results of the Kirkwood method approach those of the thin rod approximation.

It is instructive to contrast our results with those for the three-dimensional case. In three dimensions, there is a length-independent factor of two difference between the parallel drag coefficient and the perpendicular drag coefficient:

$$
\begin{aligned}
& \zeta_{\|}^{3 \mathrm{~d}}=\frac{2 \pi \eta L}{\ln \left(\frac{A L}{a}\right)} \\
& \zeta_{\perp}^{3 \mathrm{~d}}=2 \zeta_{\|}^{3 \mathrm{~d}} .
\end{aligned}
$$

The appearance of the logarithm in Eq. 26] signals the break down of a purely local drag (or, "free draining"). In other words, the long-range hydrodynamic interactions between various segments of the rod cause the drag on the rod to be reduced from simple linear dependence on $L$ as would be the case if the hydrodynamic drag on each element of the rod were purely local in character and thus the total drag additive along the length of the rod. Instead, the motion of one part of the rod sets up longranged fluid flows that effectively drag other parts of the rod forward.

The reduced dimensionality of the flow in the film qualitatively changes this result as show in Eq. 24] and figure 9 From [10 it is clear that the two drag coefficients are equal in the limit $L \ll \ell_{0}$ (the dotted line for small $L / \ell_{0}$ is simply unity) while in the limit that $L \gg \ell_{0}$ they differ substantially. In fact, as we argue here, we see an apparent, purely local drag per unit length. Hence, the ratio of the two drags for long rods is given simply by the logarithm described above, as can be seen by the asymptotic fit to a logarithm that is illustrated by the dotted line on the right of the figure.

On the one hand, while the dimensions of the rod are small $\left(\ll \ell_{0}\right)$, the dissipation is governed primarily by the film, which is insensitive to orientation and aspect ratio, just as it is to size: there is only a weak, logarithmic dependence on size in this limit [8]. On the other hand, when the rod becomes longer (than $\ell_{0}$ ), the dependence on both orientation and aspect ratio becomes stronger. Here, the three-dimensional fluid governs the dissipation, and we know that orientation and especially size matters in this limit. A major difference, however, arises when we compare parallel motion with perpendicular motion.
In the latter case, although the dissipation is governed primarily by the fluid, the film (along with its assumed incompressibility and no-slip conditions) imposes a very different boundary condition on the flow from what we would have in a bulk fluid alone. The velocity field $\vec{v}_{\perp}$ in the film satisfies $\vec{\nabla}_{\perp} \cdot \vec{v}_{\perp}=0$, which is inconsistent with the Stokes flow in perpendicular motion. Considered as a two-dimensional field, $\partial_{x} v_{x}+\partial_{y} v_{y}$ is non-zero in the plane of motion in this case. This added condition can only increase the drag for perpendicular motion relative to that without the film present. In contrast, since this boundary condition is consistent with the flow field for parallel motion, we expect to find quantitative agreement with the parallel mobility in a bulk fluid when the film's viscosity becomes irrelevant (small $\ell_{0}$ ).

For the perpendicular motion, not only is the drag increased relative to that for a bulk fluid, but the dependence is purely linear, as show in Fig. 9. The linear dependence is an indication of the absence of the hydrodynamic effects described above, which reduce the drag by cooperativity of sections along the rod. Here, the drag is simply proportional to the length of the rod. This can be seen from the additional boundary condition mentioned above. Although the dissipation for long rods is governed by the fluid viscosity, the characteristic scale for this flow is that of the whole rod. Unlike the case of perpendicular motion in a simple fluid, where there is a short path of order the rod diameter $a$ around the rod, the in-plane incompressibility forces the flows to go around the long way. Hence, the total absence of the logarithm, and the simple, purely local drag proportional to length. This can be seen in Fig. [10] where we show the ratio of the drag coefficients is just given by the logarithmic term coming from the hydrodynamics of rods in ordinary fluids. Finally, we note that these observations also explain why the rotational drag is purely algebraic for long rods, since rotations exhibit a purely local drag or rod segments perpendicular to the motion. Specifically, as shown in Fig. [11 we find

$$
\zeta_{R} \simeq 0.16 \pi \eta L^{3} .
$$

In summary we have developed a highly adaptable framework to compute the drag on irregularly shaped objects embedded in a viscous membrane or interface. As a demonstration of this method we have computed the drag on a rigid rod in this two-dimensional fluid system viscously coupled to a fluid subphase and compared our results to both the well-known results for the drag on a rod in a three-dimensional, viscous fluid, and the result for the drag on a disk embedded in a membrane (due to Saffman and Delbrück), which is applicable to e.g. the diffusion of small transmembrane proteins. Firstly, we find, in accordance with the results of previous investigators [7, 9, 10] that there is an inherent length scale $\ell$ in the system set by the ratio of the two-dimensional viscosity of the membrane to the three dimensional viscosity of the subphase fluid. The existence of such a length scale is made obvious by dimensional analysis. The importance 
of this length scale on the drag tensor associated with various objects embedded in the membrane has been discussed in this work. In brief, for objects with characteristic dimensions less than $\ell$, the Saffman-Delbrück result is recovered from our more general computation. The drag coefficient tensor for all such small objects is simply that of a small disk. It is isotropic and independent of particle size except for logarithmically small corrections. For objects significantly larger than $\ell$, our analysis shows that drag tensor becomes both size dependent and anisotropic for rod-like objects. Based on our calculations restricted to rod-like objects, it is nevertheless clear that these general statements apply to objects of arbitrary shape as well. In particular for these rods, we find that the parallel drag coefficient matches that of a rod dragged parallel to its long axis in a three-dimensional, viscous fluid. For the same rod dragged perpendicular to its length a qualitatively different result is found: the effective drag coefficient is logarithmically enhanced versus its three-dimensional counter-part due to the breakdown of long-range hydrodynamic interactions in the interface due to momentum transfer to the bulk, subphase.

While the heuristic importance of this new length scale is clear, we have not yet estimated its size for typical membranes. As a starting point, we note that if the membrane/interfacial viscous were equal to that of the bulk, subphase, this length would naturally be the thickness of the membrane, i.e. a molecular length. For a short chain surfactant monolayer or lipid bilayer this length would then be on the order of $1-2 \mathrm{~nm}$ respectively. However, it is expected that the internal viscosity of the membrane/interface is typically much larger than that of the (typically aqueous) subphase and this length $\ell$ is consequently multiplied by a factor equal to this viscosity enhancement of the membrane/interfacial material. It is not unreasonable to suppose that $\ell \sim 10-100 \mathrm{~nm}$. Thus we expect to see significant deviations from the SaffmanDelbrück result both for lipid rafts and protein aggregates that surpass such lengths. At the same time, it is clear that the standard Saffman-Delbrück result should explain the observed mobility of individual transmembrane proteins.

Further experimental tests of the above theory require the analysis of tracer particle diffusion data for a membrane/interface bound objects of various sizes. Based on these calculations, the observation of anisotropic diffusion constants for these longer rod-like objects would be a clear indication of phenomena unexplainable by the Saffman-Delbrück analysis. After testing these basic mobility calculations, one could then use these results to do both standard translational microrheology on membranes and interfaces [19] as well as novel experiments on rotational microrheology 21. Such studies will be particularly interesting in studying the properties of broken rotational phases of lipid monolayers such as hexatic phases [22]. In addition one should be able to extend the present calculations to explore the implications of membrane hydrodynamics upon diffusion limited aggregation in membranes. Such aggregation processes play an interesting role in the formation of transmembrane protein aggregates, lipid rafts, and colloidal aggregates on large, unilamellar vesicles [23].

\section{Acknowledgments}

AJL would like to thank C. Alonso and other members of the Zasadzinski group for frequent discussions. The authors acknowledge the hospitality of the Kavli Institute for Theoretical Physics where most of this work was performed. In addition AJL would like to acknowledge the hospitality of the Vrije Universiteit, Amsterdam. Finally, the authors would especially like to thank D.K. Lubensky for helpful conversations on this problem. This work is supported in part by the National Science Foundation under Grant Nos. DMR98-70785 and PHY99-07949.
[1] R.A. Stein, E.J. Hustedt, J.V. Staros, and A.H. Beth Biochem., 41, 1957 (2002).

[2] P.J.R. Spooner, R.H.E. Friesen, J. Knol, B. Poolman, and A. Watts Biophys. J., 79, 756 (2002).

[3] P. Steffen, P. Heinig, S. Worlitzer, Z. Khattariand, and T.M. Fischer J. Chem. Phys., 115, 994 (2001).

[4] J.F. Kingler and H.M. McConnell J. Phys. Chem., 97 6096 (1993).

[5] K. Simons and E. Ikonen, Nature 387, 569 (1997).

[6] A. Pralle, P. Keller, E.L. Florin, K. Simons, and J.K.H. Horder J. Cell Biol.,148, 997 (2000).

[7] B.D. Hughes, B.A. Pailthorpe, and L.R. White J. Fluid Mech., 110, 349 (1981).

[8] P.G. Saffman and M. Delbrück, P. Natl. Acad. Sci. USA 72, 3111 (1975); P.G. Saffman, J. Fluid Mech. 73, 573 (1976). See also H.A. Stone J. Fluid Mech., 409165 (2000).
[9] D.K. Lubensky and R.E. Goldstein Phys. Fluids 8,843 (1996).

[10] A. Ajdari and H.A. Stone J. Fluid Mech., 369, 151 (1998).

[11] E. Helfer, S. Harlepp, L. Bourdieu, J. Robert, F.C. MacKintosh, and D. Chatenay, Phys. Rev. Lett. 85, 457 (2000).

[12] E. Helfer, S. Harlepp, L. Bourdieu, J. Robert, F.C. MacKintosh, and D. Chatenay, Phys. Rev. E 63, 021904 (2001).

[13] E. Helfer, S. Harlepp, L. Bourdieu, J. Robert, F.C. MacKintosh, and D. Chatenay, Phys. Rev. Lett. 87, 088103 (2001).

[14] H. Lamb Hydrodynamics $6^{\text {th }}$ ed. Dover Publications, New York (1945).

[15] See, for example Chapter 8 in M. Doi and S.F. Edwards The Theory of Polymer Dynamics, Clarendon Press, Ox- 
ford (1986).

[16] Levine et al. in preparation.

[17] Private correspondance with Michael Dennin.

[18] C.F. Brooks, G.G. Fuller, C.W. Frank, and C.R. Robertson Langmuir 15, 2450 (1999); J.Q. Ding, H.E. Warriner, J.A. Zasadzinski, and D.K. Schwartz Langmuir 18, 2800 (2002).

[19] Alex J. Levine, F.C. MacKintosh, PRE 66, 061606 (2002).

[20] Handbook of Mathematical Functions with Formulas, Graphs, Mathematical Tables, eds. M. Abromowitz and
I.E. Stegun National Bureau of Standards, Washington D.C., (1964).

[21] Z. Cheng and T.G. Mason Phys. Rev. Lett.,90, 0183304 (2003).

[22] See for example: J. Ignes-Mullol and D.K. Schwartz Nature 410, 348 (2001). Also private communication with Michael Dennin.

[23] Anthony Dinsmore, private communicaiton. See also A.D. Dinsmore, M.F. Hsu, M.G. Nikolaides, M. Marquez, A.R. Bausch, and D.A. Weitz Science 298, 1006 (2002). 